\title{
Estimation-Based and Dropout-Dependent Control Design for Aeroengine Distributed Control System with Packet Dropout
}

\author{
Yun Xu $\mathbb{D}^{1},{ }^{1}$ Muxuan Pan $\left(\mathbb{D},{ }^{1}\right.$ Jinquan Huang, ${ }^{1}$ Wenxiang Zhou, ${ }^{1}$ Xiaojie Qiu, ${ }^{2}$ \\ and Ye-Hwa Chen $\mathbb{D D}^{3,4}$ \\ ${ }^{1}$ Nanjing University of Aeronautics and Astronautics, Nanjing 210016, China \\ ${ }^{2}$ Aeroengine Control Systems Institute, Wuxi 214062, China \\ ${ }^{3}$ Georgia Institute of Technology, Atlanta, GA 30332-0405, USA \\ ${ }^{4}$ Chang'an University, Xi'an 710065, China
}

Correspondence should be addressed to Muxuan Pan; muxuan.pan@nuaa.edu.cn

Received 2 November 2021; Accepted 28 January 2022; Published 21 February 2022

Academic Editor: Xingling Shao

Copyright ( 2022 Yun Xu et al. This is an open access article distributed under the Creative Commons Attribution License, which permits unrestricted use, distribution, and reproduction in any medium, provided the original work is properly cited.

A novel estimation-based and dropout-dependent control design for distributed control systems of aeroengines with packet dropout is proposed. The packet dropout is described as an independent identically distributed (i.i.d.) Bernoulli process with known probability. The objective of the control system is to effectively and stably impel aeroengines. An inverse system is proposed to reconstruct the missing measurements, based on which, a hybrid adaptive Kalman filter (HAKF) is proposed to provide the estimated states for control design. A new state-feedback control design is developed with the estimated states and the actual states. For guaranteeing the stability of aeroengines, both the stability of HAKF and the new controlled system are analyzed and proved. The new design is applied to an aeroengine, HAKF has superior estimation accuracy which ensures the effectiveness, and the desired stability performance is achieved by controlled system in the presence of packet dropout.

\section{Introduction}

As propulsion, aeroengines are a typical kind of nonlinear system with high-performance requirements. In order to meet the requirements of engine performance and efficiency, it is warranted to ameliorate the control architecture in terms of commonality, expandability, flexibility, and reduced weight [1]. At present, a very effective solution is to convert the engine centralized control system into a distributed control system (DCS). Despite the performance superiority, the distributed control system may cause packet dropout due to the characteristics of network transmission [2]. The dropout of data packets in the control system not only undermines the quality of control but also destroys the stability of the control system. It is noted that the packet dropout will lead to some technical difficulties in the stability-guaranteed control design with packet dropout. Hence, the problem of packet dropout has attracted the attention of many scholars.
Generally, the packet dropout is modelled as a random process with random Bernoulli distribution $[3,4]$ or timehomogeneous binary Markov distribution $[5,6]$ in the previous research. Both these two descriptions define the arrival of packet at instant $k$ as a binary random variable $\gamma_{k}$. In the Bernoulli process, the variable $\gamma_{k}$ was modelled with probability distribution:

$$
\begin{gathered}
P\left(\gamma_{k}=0\right)=\lambda, \\
P\left(\gamma_{k}=1\right)=1-\lambda,
\end{gathered}
$$

where the variable $\lambda$ is the packet dropout rate. In the Markov process, the arrival of a packet is featured by a transition probability matrix:

$$
M^{+}=\left(P\left\{\gamma_{k+1}=j \mid \gamma_{k}=i\right\}\right)_{i, j \in S}=\left[\begin{array}{cc}
1-q & q \\
p & 1-p
\end{array}\right],
$$


where $S=\{0,1\}$ is the state space of the Markov process and the positive parameters $p$ and $q$ are the failure rate and recovery rate, respectively. Based on these mathematical descriptions of the packet dropout process, the compensation or estimation for the lost information is developed simultaneously.

Considering the information compensation for packet dropout, researchers have proposed numerous and outstanding results of estimation approaches with intermittent observations. Among these approaches, the Kalman filter $(\mathrm{KF})$ has received a great of attention because it is a minimum mean-square error (MMSE) estimation under the packet dropout conditions. Sinopoli proposed the Kalman state estimator by introducing the random binary variable $\gamma_{k}$ of a Bernoulli packet dropout into the algorithm iteration and proved the stability of the Kalman estimator [7]. Based on the Kalman iteration structure proposed in reference [7], a series of research on the stability of new Kalman filters is developed and published. Reference [8] introduces the peak covariance to describe the stability of the Kalman filter with the stochastic packet dropout and obtains the stability conditions. An improved stability condition for the Kalman filter with bounded packet dropout was presented in reference [9]; a less conservative and sufficient condition for peak-covariance stability is established by the linear matrix inequality (LMI) method. Furthermore, the peakcovariance stability of Kalman filters is proven to be the mean-square stability for a random packet dropout [10, 11]. These theories extend to the analysis of Kalman filters with complicated systems [12-16]. The design of Kalman filters with state-dependent packet dropout for the hybrid measurement system is investigated in reference [12]. Reference [13] proposed a specific estimation design with Markov packet dropout for nonuniform sampling network systems. Marelli analyzed the stability for Kalman filters with random measurement matrices, the necessary and sufficient conditions are given in reference [14]. An extension of the fruits of [14] is made in reference [15] to demonstrate the stability of multisensor Kalman filter over a lossy network. Similarly, the stability analysis of multisensor Kalman filter over lossy networks is also discussed in reference [16]. From all the above references, the Kalman filter supplies a solution for the packet dropout problem, which can compensate for the losing information effectively. In this way, the construction of an estimator can serve as the basis of information compensation with packet dropout.

Due to the control system performance affecting the safety of aeroengine directly, the stability is the primary and most important requirement of the controller design for aeroengines. To guarantee the safety of aeroengine, efforts should be devoted to the controller stability analysis with packet dropout. In the stability with network failure literature, the classical approach is to consider network failure as a disturbance and utilize the Lyapunov theorem to analyze the controller stability [17-21], and then, these authors take advantage of the controller robustness to cover the poor effect. Moreover, the other arguably popular problem is the controller design with unmeasurable states and packet drop- out. To identify the unmeasurable states, observers are constructed and the observer-based controllers come after [22-24]. Similarly, the observer-based control system framework is also used for the packet dropout and the relative system stability analysis is researched. Wang et al. designed an observer for the unmeasurable states and achieve the exponentially mean-square stability condition for the observerbased control system [25]. Li et al. proposed a stability analysis approach for the network nonlinear system with global Lipschitz nonlinearities and random packet dropout [26]. Similarly, the interesting publications of the observer-based control system with packet dropout and other problems (such as consecutive packet delays, multiplicative noises, and uncertainties) are investigated in references [19-21, 27]. Furthermore, some new control designs for the system with network failure are proposed [28-30]. The novel control design is attracted to the event-triggered mechanism, which can guarantee the control system stability and reduce the network burden. These studies supply a new direction for solving the network failure problems by the codesign of the control and scheduling approach.

The abovementioned outstanding researches mainly focus on the control design for the system with network failure, robust control, observer-based control, and codesign of control and scheduling approaches are investigated. Actually, the network failure in aeroengine DCS would cause the loss of controller inputs which is the crucial factor for the aeroengine control system. Hence, it is necessary to compensate for the missing controller inputs for the aeroengine. To guarantee the performances of aeroengine DCS, the packet dropout for the specific aeroengine DCS is handled by a separated design framework which consists of the estimation and estimation-based control design, which is quite different from the above research in theory [17-21, 27-30].

The traditional Kalman filters are widely used for estimation. And numerous researches on the design and stability analysis of the Kalman filter with packet dropout were carried out. It is noted that the Kalman structure in the above references gives up the measurement update, an important correct step, due to the measurement dropout. And this way would directly decrease the estimation accuracy. Besides, the stability analysis of both the Kalman filters and the control system are separated; there is a gap in the connection between information compensation and control system stability. Considering aeroengines are specific time-varying, a complex controlled plant with strong nonlinearity requires the high control performance and quality. It is necessary to combine the information compensation and control system stability analysis for the high requirements of the aeroengines.

For the purpose to shorten the gap mentioned above, unlike the popular state estimation methods (traditional $\mathrm{KF}$, see, e.g., references $[7,8])$, we proposed a hybrid adaptive Kalman filter (HAKF), an approach with hybrid measurement correct based on the Kalman structure, to increase the estimation accuracy with packet dropout. The main difference between HAKF and the above Kalman filters is that there is a measurement reconstruction step in ours which improves the accuracy. Moreover, the stability of the aeroengine control system with HAKF which incorporates 
information compensation into the control system stability is investigated. The corresponding stability conditions of this control system were presented in the formulation of linear matrix inequalities (LMIs).

The salient novelties and contributions in this paper are as follows:

(1) The new approach deals with packet dropout and the control design separately, which significantly improves the flexibility of the control system design and boosts the performance of the control system. In theory, the novel estimator effectively compensates the missing states, and the controller guarantees the desired stability performance

(2) The inverse system combined with references reconstructs the missing measurable information. The reconstructed information serves as the basis of the measurement update in state estimation and improves the accuracy of the new estimation

(3) By taking advantage of the reconstructed information, the measurement, and the packet dropout flag $\gamma_{k}$, the HAKF improves the estimation accuracy obviously, which is distinctly different from the traditional Kalman filters [7, 8]. Moreover, the stability of the HAKF is guaranteed in theory

(4) The proposed control design employs the hybrid information, namely, actual and estimated information. Furthermore, by a new mathematical expectation-based Lyapunov function, the stability of this hybrid control system is guaranteed

(5) The new approach is applied to a turbofan engine. The results demonstrate that the HAKF decreases the RMSEs of the estimation by over $57 \%$ compared to the traditional Kalman filters, and the control system has superior performances with the proposed control

This paper is organized as follows. In Section 2, the mathematical description of the aeroengine distributed control system with packet dropout is formulated. Section 3 proposes the design and stability analysis of the HAKF. Section 4 presents the dropout-dependent stability analysis of the hybrid signal closed-loop control system. In Section 5, the numerical examples are given to illustrate the comparison of novel HAKF and the traditional filter in references [7, 8]; besides, the stability simulations on the aeroengine are also described. Some conclusions of this paper are drawn finally.

\section{Problem Formulation}

The aeroengine system is described as

$$
\left\{\begin{array}{l}
x_{k+1}=A x_{k}+B u_{k}+w_{k}, \\
y_{k}=C x_{k}+D u_{k}, \\
z_{k}=W x_{k}+v_{k},
\end{array}\right.
$$

where $x_{k} \in \mathbb{R}^{n}$ and $u_{k} \in \mathbb{R}^{m}$ are the state and control inputs, $y_{k} \in \mathbb{R}^{p}$ is the output, $z_{k} \in \mathbb{R}^{l}$ is the measurement, and $w_{k}$ $\in \mathbb{R}^{n}$ and $v_{k} \in \mathbb{R}^{l}$ are the system and measurement noises. The matrices $A \in \mathbb{R}^{n \times n}, B \in \mathbb{R}^{n \times m}, C \in \mathbb{R}^{p \times n}, D \in \mathbb{R}^{p \times m}$, and $W \in \mathbb{R}^{l \times n}$ are known. The noises $w_{k}$ and $v_{k}$ are Gaussian noise with

$$
\begin{gathered}
E\left[w_{k}\right]=0, E\left[v_{k}\right]=0, \\
E\left[w_{k} w_{k}^{T}\right]=Q_{k}, E\left[v_{k} v_{k}^{T}\right]=R_{k} .
\end{gathered}
$$

To improve the aeroengine controller performance and design flexibility, an estimation-based control framework for aeroengine DCS is shown in Figure 1. Data packets are transmitted from sensors to the controller and then to the actuators via a shared network in the DCS. Inevitably, a sophisticated packet may dropout in the data transmission.

Assumption 1. For the aeroengine DCS with stochastic packet dropout, the following assumptions are introduced:

(1) Sensors are time-triggered, and the controller is event-triggered

(2) The sensor data is sent following a sequence of sampling

(3) The initial states transmitted from the controller to the actuator are consistent at the initial moment

(4) The actuators take advantage of the data received in the latest previous sample when the packet dropout

Remark 2. The data packets may dropout in the transmission from sensors to controller or/and from the controller to actuators. Assumption 1 provides a strategy to compensate for the lost information between the controller and actuators based on the time-triggered network transmission protocol. While the packet dropout between the sensors and controller, it would cause the performance deterioration of the controlled system (3). Hence, this paper focuses on compensation and control with problem of the missing data packet.

Due to the uncertainties of the external environment, network characteristics, etc., the packet dropout is commonly designed as a random process. The packet dropout in the network channel is considered a statistic random process with multicycle information missing because of the stochastic and disordered uncertainties. Define a random variable $\gamma_{k}$ to describe whether the controller receives the observations at the sample instant $k$ [31-33], and it is formulated as follows:

$$
P\left(\gamma_{k}=0\right)=\lambda, \gamma_{k} \in\{0,1\} .
$$

Here, $\gamma_{k}$ is a binary random variable subject to independent identically distributed (i.i.d.) process. The variable $\gamma_{k}$ $=1$ indicates that the controller accepts the sensor signals successfully and there are no data dropout at instant $k$. 


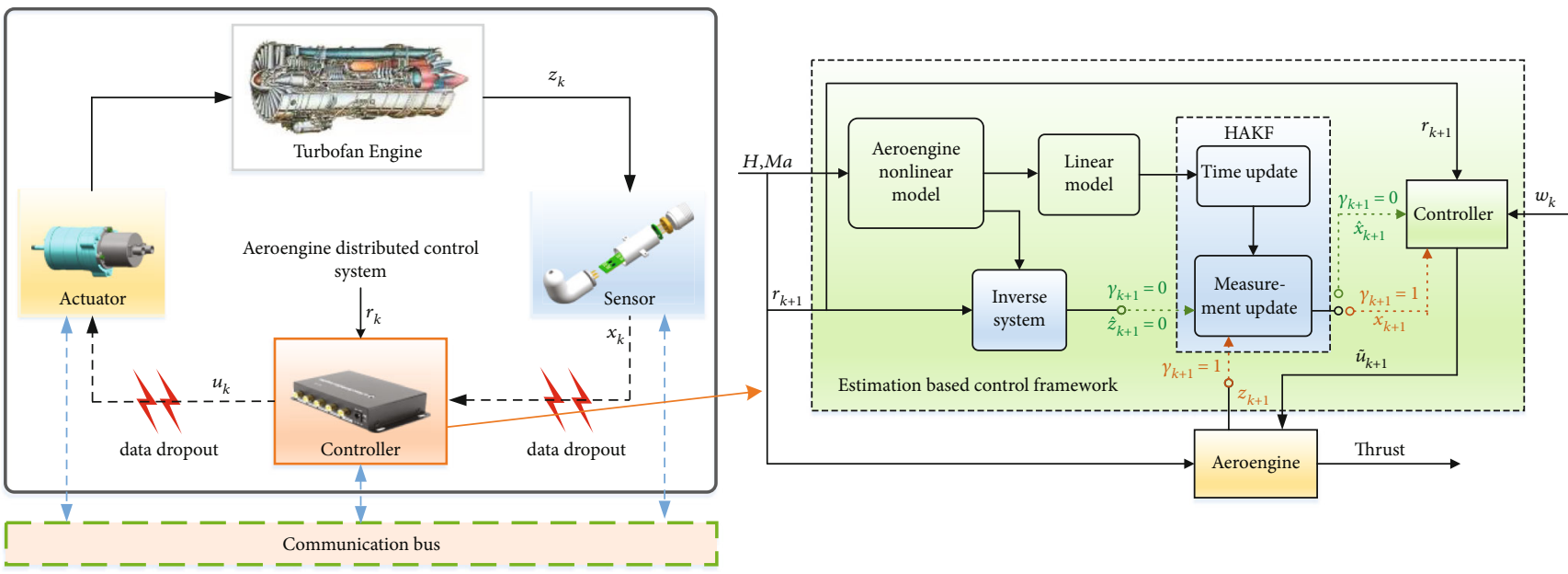

FIGURE 1: Framework of aeroengine DCS with estimation-based control approach.

Using $\gamma_{k}=0$ demonstrates that the controller fails to accept the sensor signals and the packet lost. Therefore, the network transmission process in an aeroengine DCS can be shown as Figure 2, in which $\tilde{z}_{k}$ is actual information that the controller can receive at instant $k$.

$$
\tilde{z}_{k}=\gamma_{k} z_{k}
$$

From (7), when $\gamma_{k}=0$, the information for controller was lost, which immediately destroy the controller quality and performance. Considering the information compensation, an estimator is designed as a guard for the controller and the novel controller framework with signal transmission is shown in Figure 3.

\section{Hybrid Adaptive Kalman Filter}

3.1. Structure of the HAKF. In view of the system (3) with packet dropout, we propose the hybrid adaptive Kalman filter (HAKF) to compensate for the missing information.

The HAKF time update is

$$
\begin{gathered}
\widehat{x}_{k+1}^{-}=A \widehat{x}_{k}+B u_{k}, \\
P_{k+1}^{-}=E\left[e_{k+1}^{-} e_{k+1}^{-}{ }^{T}\right]=A P_{k} A^{T}+Q_{k},
\end{gathered}
$$

and the posteriori estimated state is

$$
\begin{gathered}
\hat{x}_{k+1}=\widehat{x}_{k+1}^{-}+K_{k+1}\left(\tilde{z}_{k+1}-W \hat{x}_{k+1}^{-}\right), \\
P_{k+1}=E\left[e_{k+1} e_{k+1}^{T}\right],
\end{gathered}
$$

where $\widehat{x}_{k} \in \mathbb{R}^{n}$ is the estimated state at instant $k, \widehat{x}_{k+1}^{-}$and $\widehat{x}_{k+1} \in \mathbb{R}^{n}$ are the priori and posteriori estimated states, and $e_{k+1}^{-}$and $e_{k+1} \in \mathbb{R}^{n}$ are the priori and posteriori estimation errors with

$$
e_{k+1}^{-}=\widehat{x}_{k+1}^{-}-x_{k+1}, e_{k+1}=\widehat{x}_{k+1}-x_{k+1},
$$

$P_{k+1}^{-}$and $P_{k+1} \in \mathbb{R}^{n \times n}$ are the priori estimation error covariance and the posteriori estimation error covariance, $K_{k+1} \in \mathbb{R}^{n \times l}$ is the gain of HAKF, and $\tilde{z}_{k+1} \in \mathbb{R}^{l}$ is the hybrid measurement.

Remark 3. The HAKF time update (8) and (9) supply the priori estimated state, and then, the correct module (10) was designed to update the priori estimated state. In view of the time update, equations (8) and (9) are just model based which are irrelevant to the measurements; the priori estimated state and priori error covariance matrix of HAKF are regular as the LKF as shown in [34].

Remark 4. According to the LKF theory, the adjustment of the KF estimation state was motivated by the measurement errors $\tilde{z}_{k+1}-W \hat{x}_{k+1}^{-}$. Equation (7) shows that no measurement is included when a packet dropout and the traditional KF has no correct module to update the priori estimation [7, $8]$. When a continuous package dropout occurs, the accuracy of the KF decreases significantly. In practice, the packet dropout comes up occasionally, which means the controller can receive the measurements when the network works. Hence, the HAKF adaptively adopts measurements or reconstructed measurements when the network works or package dropout occurs.

Remark 5. The key and primary difficulty is to design an approach to reconstruct the missing measurements for the HAKF. In Section 3.2, this difficult is addressed by the measurement reconstruction with an inverse system.

3.2. Measurement Reconstruction. The meaningless measurements need to be reconstructed when $\gamma_{k}=0$, inspired by the intelligent inverse control [35], we reconstruct the measurement by combination of inverse system and references. The inverse system connects with a previous system in a series is shown in Figure 4, which can distinctly reveal the relationship between inverse system $S_{L}^{-1}$ and the original system $S$. 


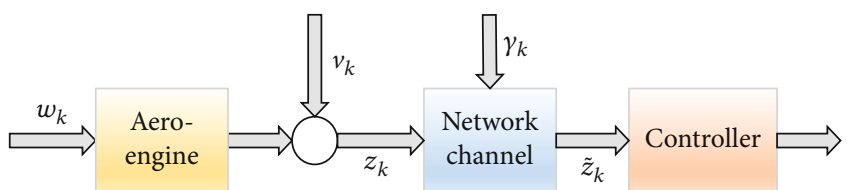

Figure 2: Network transmission process.

Considering system (3) at the instant $k+1$, its inputoutput system is

$$
y_{S}=S u_{S}
$$

Here, $u_{S}=\left[\begin{array}{ll}x_{k+1}^{T} & u_{k+1}^{T}\end{array}\right]^{T} \in \mathbb{R}^{n+m}, \quad y_{S}=\left[x_{k+2}^{T} y_{k+1}^{T}\right]^{T} \in \mathbb{R}^{n+p}$, and $S=\left[\begin{array}{cc}A & B \\ C & D\end{array}\right] \in \mathbb{R}^{(n+p) \times(n+m)}$. The subscript $k+2$ means one step after $k+1$.

The inverse system of (13) is defined as

$$
\widehat{u}_{k+1}=S_{L}^{-1} \widehat{y}_{k+1}
$$

here, $\widehat{u}_{k+1}=\left[\begin{array}{ll}\widehat{x}_{k+1}^{T} & \widehat{u}_{k+1}^{T}\end{array}\right]^{T}, \widehat{y}_{k+1}=\left[x_{k+2}^{T} r_{k+1}^{T}\right]^{T} \in \mathbb{R}^{n+p}, \quad r_{k+1}$ is the reference, and $S_{L}^{-1} \in \mathbb{R}^{(n+m) \times(n+p)}$ satisfying $S_{L}^{-1} S=$ $I_{(n+m) \times(n+m)}$.

Remark 6. In an aeroengine closed-loop tracking control system such as Figure 1, the ideal system response performances are outputs $y_{k+1}$ tracking for the reference signals $r_{k+1}$ without steady errors, and this performance can be achieved by controller design, a tracking error-driven feedback approach [35]. Based on this characteristic, we reconstruct the sensor signals $\widehat{z}_{k+1}$ by the aeroengine inverse system $S_{L}^{-1}$ combined with the references $r_{k+1}$, and $\widehat{x}_{k+1}$ would be equal to the actual $x_{k+1}$ with the assumption of no tracking errors.

Based on the inverse system (14), we reconstruct the output as

$$
\widehat{z}_{k+1}=W\left[I_{n \times n} \quad 0_{n \times m}\right] S_{L}^{-1} \widehat{y}_{k+1}=W \widehat{x}_{k+1} .
$$

Finally, the receiving measurements $\tilde{z}_{k+1}$ can be written as

$$
\tilde{z}_{k+1}=\gamma_{k+1} z_{k+1}+\left(1-\gamma_{k+1}\right) \widehat{z}_{k+1} \text {. }
$$

Remark 7. By introducing the packet dropout variable $\gamma_{k+1}$, equation (16) merges the measurement $z_{k+1}$ and its reconstructed value $\widehat{z}_{k+1}$ to $\tilde{z}_{k+1}$. When the network transmission works, $\tilde{z}_{k+1}=z_{k+1}$, while the packet dropout occurs, $\tilde{z}_{k+1}=$ $\widehat{z}_{k+1}$, which can effectively rectify the abandoned correct module in references $[7,8]$.

It is noted that the HAKF gain $K_{k+1}$ in (10) will affect the posteriori estimation error $e_{k+1}$ defined in (12). So, in the next subsection, we present the optimization of $K_{k+1}$ to minimize the error $e_{k+1}$.

3.3. Design of HAKF Gain Matrix. Define the performance function as

$$
J\left(K_{k+1}\right)=\min _{K_{k+1}} \sum_{i=1}^{n} e_{k+1}^{2}(i)
$$

here, $e_{k+1}(i)$ is the $i$ th entry of $e_{k+1}$. Therefore, the performance function $J\left(K_{k+1}\right)$ can be regarded as

$$
J\left(K_{k+1}\right)=\min _{K_{k+1}} T_{r}\left(P_{k+1}\right),
$$

where $T_{r}(\cdot)$ is the trajectory of a matrix $[34,36]$.

Introducing equations (10) and (16) into (12), it yields

$$
e_{k+1}=\widehat{x}_{k+1}-x_{k+1}=\widehat{x}_{k+1}^{-}+K_{k+1}\left(\tilde{z}_{k+1}-W \widehat{x}_{k+1}^{-}\right)-x_{k+1}
$$

where $v_{\mathrm{k}+1}$ is the residual of HAKF with

$$
\begin{aligned}
v_{k+1}= & \left(\gamma_{k+1} z_{k+1}+\left(1-\gamma_{k+1}\right) \widehat{z}_{k+1}\right)-W \widehat{x}_{k+1}^{-}=e_{k+1}^{-} \\
& +K_{k+1}\left(\left(\gamma_{k+1} z_{k+1}+\left(1-\gamma_{k+1}\right) \widehat{z}_{k+1}\right)-W \widehat{x}_{k+1}^{-}\right) \\
= & e_{k+1}^{-}+K_{k+1} v_{k+1}
\end{aligned}
$$

By formulas (3) and (15) and Remark 6, it follows:

$$
\begin{aligned}
v_{k+1} & =\left(\gamma_{k+1} z_{k+1}+\left(1-\gamma_{k+1}\right) \widehat{z}_{k+1}\right)-W \widehat{x}_{k+1}^{-} \\
& =-W e_{k+1}^{-}+\gamma_{k+1} v_{k} .
\end{aligned}
$$

By formula (21), the error $e_{k+1}$ in (19) can be rewritten as

$$
\begin{aligned}
e_{k+1} & =e_{k+1}^{-}+K_{k+1}\left(-W e_{k+1}^{-}+\gamma_{k+1} v_{k}\right) \\
& =\left(I-K_{k+1} W\right) e_{k+1}^{-}+\gamma_{k+1} K_{k+1} v_{k},
\end{aligned}
$$

and then, $P_{k+1}$ in equation (11) is

$$
\begin{aligned}
P_{k+1}= & \left(I-K_{k+1} W\right) E\left[e_{k+1}^{-} e_{k+1}^{-T}\right]\left(I-K_{k+1} W\right)^{T} \\
& +\gamma_{k+1}\left(I-K_{k+1} W\right) E\left[e_{k+1}^{-} v_{k}^{T} K_{k+1}^{T}\right] \\
& +\gamma_{k+1}\left(I-K_{k+1} W\right)^{T} E\left[K_{k+1} v_{k} e_{k+1}^{-T}\right] \\
& +\gamma_{k+1}{ }^{2} K_{k+1} E\left[v_{k} v_{k}^{T}\right] K_{k+1}^{T} .
\end{aligned}
$$

Since the measurement noises $v_{k}$ are uncorrelated to the priori estimated error $e_{k+1}^{-}$[37], equation (23) can be simplified as

$$
\begin{aligned}
P_{k+1}= & \left(I-K_{k+1} W\right) E\left[e_{k+1}^{-} e_{k+1}^{-}\right]\left(I-K_{k+1} W\right)^{T} \\
& +\gamma_{k+1}{ }^{2} K_{k+1} E\left[v_{k} v_{k}^{T}\right] K_{k+1}^{T} .
\end{aligned}
$$

By equations (5) and (9), it follows

$$
P_{k+1}=\left(I-K_{k+1} W\right) P_{k+1}^{-}\left(I-K_{k+1} W\right)^{T}+\gamma_{k+1}{ }^{2} K_{k+1} R_{k} K_{k+1}{ }^{T} .
$$




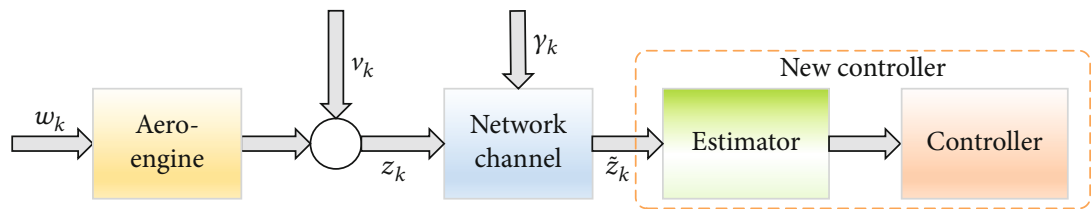

FIGURE 3: Network transmission with estimator.

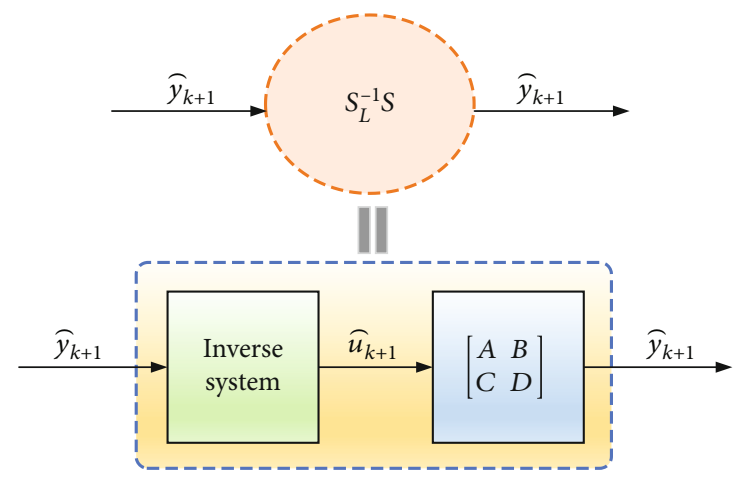

FIgURE 4: Inverse system of an aeroengine system.

To solve (18), the partial differential to $K_{k+1}$ is

$$
\begin{aligned}
\frac{\partial\left(J\left(K_{k+1}\right)\right)}{\partial K_{k+1}} & =-2 P_{k+1}^{-} W^{T}+2 K_{k+1} W P_{k+1}^{-} W^{T}+2 \gamma_{k+1}^{2} K_{k+1} R_{k} \\
& =-2\left(I-K_{k+1} W\right) P_{k+1}^{-} W^{T}+2 \gamma_{k+1}^{2} K_{k+1} R_{k} .
\end{aligned}
$$

Let $\partial\left(J\left(K_{k+1}\right)\right) / \partial K_{k+1}=0[38,39]$, and it yields

$$
K_{k+1}=P_{k+1}^{-} W^{T}\left(W P_{k+1}^{-} W^{T}+\gamma_{k+1}^{2} R_{k}\right)^{-1} .
$$

Finally, considering system (3) with the reconstructed measurements (16), by equations (8)-(10), (25), and (27), the HAKF state estimation approach is summarized as

$$
\begin{gathered}
\hat{x}_{k+1}^{-}=A \widehat{x}_{k}+B u_{k}, \\
P_{k+1}^{-}=E\left[e_{k+1}^{-} e_{k+1}^{-T}\right]=A P_{k} A^{T}+Q_{k}, \\
\widehat{x}_{k+1}=\widehat{x}_{k+1}^{-}+K_{k+1}\left(\tilde{z}_{k+1}-W \widehat{x}_{k+1}^{-}\right), \\
P_{k+1}=\left(I-K_{k+1} W\right) P_{k+1}^{-}\left(I-K_{k+1} W\right)^{T}+\gamma_{k+1}^{2} K_{k+1} R_{k} K_{k+1}^{T}, \\
K_{k+1}=P_{k+1}^{-} W^{T}\left(W P_{k+1}^{-} W^{T}+\gamma_{k+1}^{2} R_{k}\right)^{-1} .
\end{gathered}
$$

3.4. HAKF Design Procedure. The design procedure of HAKF is summarized as follows, and the iteration steps of the new estimation approach are shown in Figure 5.

Step 1. Set up the initial state values for $\widehat{x}_{0}, u_{0}, \widehat{P}_{0}$ and the noise and system matrix $Q_{k}, R_{k}, A, B, C, D$, and $W$

Step 2. Calculate the prior estimated state $\widehat{x}_{k+1}^{-}$and prior estimation error covariance $P_{k+1}^{-}$at instant $k+1$ based on (28) and (29) with the state variable $\widehat{x}_{k}$ and input variable $u_{k}$ at instant $k$

Step 3. Determine the situation of network channel and reconstruct the measurement signals $\tilde{z}_{k+1}$ based on (15) and (16) by the binary variable $\gamma_{k+1}$

Step 4. Calculate the Kalman gain $K_{k+1}$ based on (32)

Step 5. Update the posterior state $\widehat{x}_{k+1}$ with (30) by measurement information $\tilde{z}_{k+1}$ and calculate the posterior estimation error covariance $P_{k+1}$ based on (31)

Step 6. Output $\widehat{x}_{k+1}, P_{k+1}$, and update the time instant.

3.5. Stability Analysis of HAKF with Packet Dropout. The proposed HAKF (28)-(32) takes advantage of the reconstructed measurement (16), which results in the additional estimated deviation. In this subsection, we present the following main result to investigate the stability of the new estimation algorithm.

Theorem 8. Consider the system (3) with packet dropout (4). If the matrices $Q_{k} \geq 0$ and $R_{k}>0$, the HAKF (28)-(32) is stable.

Proof. Consider the Lyapunov function candidate

$$
V_{k}=e_{k}^{T} P_{k}^{-1} e_{k},
$$

for any arbitrary $e_{k} \neq 0, V_{k}>0$. Let

$$
\Delta V_{k+1}=V_{k+1}-V_{k}=e_{k+1}^{T} P_{k+1}^{-1} e_{k+1}-e_{k}^{T} P_{k}^{-1} e_{k} .
$$

By (3), (10), and (12), we have

$$
\begin{aligned}
e_{k+1}= & \widehat{x}_{k+1}-x_{k+1}=\left(A-K_{k+1} W A\right) e_{k} \\
& +\left(\gamma_{k+1} K_{k+1} W-I\right) w_{k}+\gamma_{k+1} K_{k+1} v_{k} .
\end{aligned}
$$

Define

$$
F=\gamma_{k+1} K_{k+1} W-I, G=\gamma_{k+1} K_{k+1} .
$$

By formulas (34)-(36), it follows

$$
\begin{aligned}
\Delta V_{k+1}= & {\left[\left(A-K_{k+1} W A\right) e_{k}\right]^{T} P_{k+1}^{-1}\left[\left(A-K_{k+1} W A\right) e_{k}\right] } \\
& +\left[\left(A-K_{k+1} W A\right) e_{k}\right]^{T} P_{k+1}^{-1}\left(F w_{k}+G v_{k}\right) \\
& +\left(F w_{k}+G v_{k}\right)^{T} P_{k+1}^{-1}\left[\left(A-K_{k+1} W A\right) e_{k}\right] \\
& +\left(F w_{k}+G v_{k}\right)^{T} P_{k+1}^{-1}\left(F w_{k}+G v_{k}\right)-e_{k}^{T} P_{k}^{-1} e_{k} .
\end{aligned}
$$




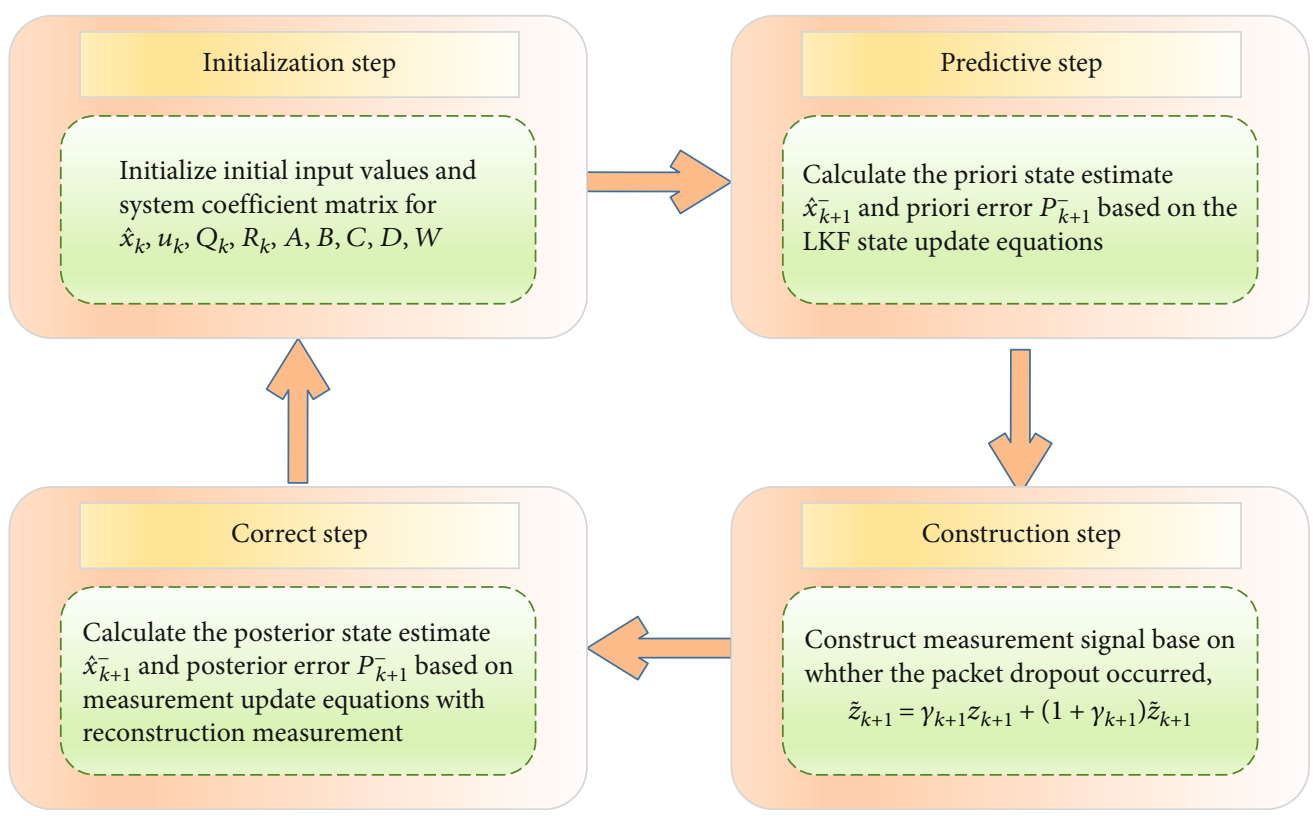

Figure 5: Iteration steps of HAKF.

Since the noise $v_{k}$ always exists, $e_{k}$ cannot decay to 0 at the end; the best hope is the homogeneous part of (35) must decay with time. Considering the dynamics of (35) is explicitly irrelevant to the noises $[40,41]$, the stability condition (37) can be simplified as

$$
\Delta V_{k+1}=e_{k}^{T}\left[\left(A-K_{k+1} W A\right)^{T} P_{k+1}^{-1}\left(A-K_{k+1} W A\right)-P_{k}^{-1}\right] e_{k} .
$$

Define

$$
\begin{gathered}
C_{M}=\left(A-K_{k+1} W A\right)^{T} P_{k+1}^{-1}\left(A-K_{k+1} W A\right)-P_{k}^{-1}, \\
\left\{\begin{array}{l}
\Phi=\left[\left(A-K_{k+1} W A\right)^{T} P_{k+1}^{-1}\right]^{-1}, \\
\Pi=P_{k} A^{T}\left(I-K_{k+1} W\right)^{T} .
\end{array}\right.
\end{gathered}
$$

Premultiply and postmultiply equation (39) by $\Phi$ and $\Pi$, and we have

$$
\Phi C_{M} \Pi=\left(I-K_{k+1} W\right) A P_{k} A^{T}\left(I-K_{k+1} W\right)^{T}-P_{k+1},
$$

by introducing equations (9) and (25) into (41), we have

$$
\begin{aligned}
\Phi C_{M} \Pi= & \left(I-K_{k+1} W\right) A P_{k} A^{T}\left(I-K_{k+1} W\right)^{T} \\
& -\left(I-K_{k+1} W\right)\left(A P_{k} A^{T}+Q_{k}\right)\left(I-K_{k+1} W\right)^{T} \\
& -\gamma_{k+1}^{2} K_{k+1} R_{k} K_{k+1}^{T}=-\left(I-K_{k+1} W\right) Q_{k}\left(I-K_{k+1} W\right)^{T} \\
& -\gamma_{k+1}^{2} K_{k+1} R_{k} K_{k+1}^{T} .
\end{aligned}
$$

As $\gamma_{k}$. is a binary random variable subject to the i.i.d. process, $Q_{k} \geq 0$ and $R_{k}>0, \Phi C_{M} \Pi \leq 0$ and $\Delta V_{k+1} \leq 0$. Hence, the Lyapunov function $V_{k}$ is negative.
Theorem 8 is proved. $\square$

Remark 9. Because the package dropout rate $\lambda$ is not $100 \%$, the binary random $\gamma_{k+1}$ is 1 with the probability of $1-\lambda$. Hence, $\Delta V_{k+1}$ and $\Delta V_{k+1}$ are negative with the same probability and $V_{k}$ will damp with the time.

Remark 10. Taking the advantage of $\gamma_{k+1}$, the HAKF renders the state estimation to adapt to the network environment, which characterize the new approach distinctly different from the LKF.

Considering the existence estimation error of the HAKF algorithm and for the purpose of improving system performance, two states are in the aeroengine closed-loop control system. First, when the controller received the measurement information successfully, the actual system states are contributed to design the controller. Otherwise, the inputs of the controller are estimated state by the HAKF with measurement reconstruction. It is worth noting that the state estimator iteratively computes at each sample time, while the inputs of controller vary with the random variable $\gamma_{k}$.

\section{Dropout-Dependent Stability Analysis for Control System}

In Section 3, the stability of HAKF is discussed. In this section, we further investigate the dropout-dependent stability of this controlled system.

We consider the controlled system (3) with the state estimation of HAKF. As shown in Figure 1, to reduce the influence of the estimation error, the controller receives the actual state $x_{k}$ when $\gamma_{k}=1$ and utilizes the estimated state $\widehat{x}_{k}$ while $\gamma_{k}=0$. Therefore, the closed-loop control system 
(3) with hybrid signals can be described as

$$
\tilde{x}_{k+1}=\tilde{A} \tilde{x}_{k}+\tilde{B} \tilde{u}_{k}+\tilde{w}_{k}
$$

where the system hybrid state $\tilde{x}_{k} \in \mathbb{R}^{2 n}$ and input $\tilde{u}_{k} \in \mathbb{R}^{2 m}$ are

$$
\tilde{x}_{k}=\left[\begin{array}{ll}
x_{k}^{T} & x \wedge_{k}^{T}
\end{array}\right]^{T}, \tilde{u}_{k}=\left[\begin{array}{ll}
u_{k}^{T} & u \wedge_{k}^{T}
\end{array}\right]^{T},
$$

where $\widehat{u}_{k} \in \mathbb{R}^{m}$ is the control variable calculated by $\widehat{x}_{k}$ and $\tilde{A}$ and $\tilde{B}$ are matrices with appropriate dimension. State equation (43) simply reveals the relationship of state at time $k$ and $k+1$, but it is worth paying attention that, because of the estimated state in the system (43), the system process noise variable $\tilde{w}_{k}$ is not as same as $w_{k}$ in (3).

Remark 11. Since the two states switch in the system (43) at each sample instant $k$, there are challenges to the stability of the control design. The next stability analysis will respond to the challenges.

In system (43), the four cases of state at instant $k+1$ come after.

Case 1. The system information is missing at both instants $k$ and $k+1$, and there is

$$
\left\{\begin{array}{l}
\gamma_{k}=0, \gamma_{k+1}=0 \\
\widehat{x}_{k+1}=A \widehat{x}_{k}+B \widehat{u}_{k}+K_{k+1}\left(\widehat{z}_{k+1}-W \widehat{x}_{k+1}^{-}\right) .
\end{array}\right.
$$

Case 2. The system information was sent successfully at instant $k$, while it is missing at instant $k+1$. There is

$$
\left\{\begin{array}{l}
\gamma_{k}=1, \gamma_{k+1}=0, \\
\widehat{x}_{k+1}=A \widehat{x}_{k}+B u_{k}+K_{k+1}\left(\widehat{z}_{k+1}-W \widehat{x}_{k+1}^{-}\right) .
\end{array}\right.
$$

Case 3. The system information is missing at instant $k$, while it was sent successfully at instant $k+1$. There is

$$
\gamma_{k}=0, \gamma_{k+1}=1, x_{k+1}=A \widehat{x}_{k}+B \widehat{u}_{k}+w_{k} \text {. }
$$

Case 4. The system information is sent successfully at both instants $k$ and $k+1$, there is

$$
\gamma_{k}=1, \gamma_{k+1}=1, x_{k+1}=A x_{k}+B u_{k}+w_{k} \text {. }
$$

Considering these four cases, the state in (44) can be further written as

$$
\begin{gathered}
x_{k+1}=\gamma_{k+1}\left(1-\gamma_{k}\right)\left(A \widehat{x}_{k}+B \widehat{u}_{k}\right)+\gamma_{k+1} \gamma_{k}\left(A x_{k}+B u_{k}\right)+\gamma_{k+1} w_{k}, \\
\widehat{x}_{k+1}=\left(1-\gamma_{k+1}\right) A x_{k}+\left(1-\gamma_{k+1}\right)\left(1-\gamma_{k}\right) B \widehat{u}_{k}+\left(1-\gamma_{k+1}\right) \gamma_{k} B u_{k} .
\end{gathered}
$$

The matrices and the noise $\tilde{w}_{k}$ in the system (43) can be explicated as

$$
\left\{\begin{array}{l}
\tilde{A}=\left[\begin{array}{cc}
\gamma_{k+1} \gamma_{k} & \gamma_{k+1}\left(1-\gamma_{k}\right) \\
\left(1-\gamma_{k+1}\right) & 0
\end{array}\right] A, \\
\tilde{B}=\left[\begin{array}{cc}
\gamma_{k+1} \gamma_{k} & \gamma_{k+1}\left(1-\gamma_{k}\right) \\
\left(1-\gamma_{k+1}\right) \gamma_{k} & \left(1-\gamma_{k+1}\right)\left(1-\gamma_{k}\right)
\end{array}\right] B, \\
\tilde{w}_{k}=\left[\begin{array}{c}
\gamma_{k+1} w_{k} \\
0
\end{array}\right] .
\end{array}\right.
$$

Let the state feedback control law is

$$
\tilde{u}_{k}=K_{X} \tilde{x}_{k}
$$

Introduce equation (51) into (43), and we have

$$
\tilde{x}_{k+1}=\left(\tilde{A}+\tilde{B} K_{X}\right) \tilde{x}_{k}+\tilde{w}_{k}
$$

Lemma 12. (Schur complement, see [42]). For a symmetric matrix $L=L^{T}=\left[\begin{array}{cc}L_{11} & L_{12} \\ * & L_{22}\end{array}\right], L_{11} \in \mathbb{R}^{r \times r}$, the following statements are equivalent:

$$
\begin{gathered}
L<0, \\
L_{11}<0, L_{22}-L_{12}^{T} L_{11}^{-1} L_{12}<0, \\
L_{22}<0, L_{11}-L_{12} L_{22}^{-1} L_{12}^{T}<0 .
\end{gathered}
$$

Lemma 13. Consider a given discrete-time system:

$$
\left\{\begin{array}{l}
x_{k+1}=f\left(x_{k}, t\right) \\
f\left(x_{e}, t\right)=0, \forall t
\end{array}\right.
$$

If there exists a positive definite function $V(\bullet) \in \mathbb{R}^{n} \times \mathbb{R}$ $\longrightarrow \mathbb{R}_{+}$, for any $k \in \mathbb{N}$ and $x_{k} \neq 0 \in \mathbb{R}^{n}$ such that

$$
\Delta V\left(x_{k}, k\right)=V\left(x_{k+1}, k+1\right)-V\left(x_{k}, k\right)<0,
$$

system (54) is asymptotically stable.

Theorem 14. Consider a closed-loop state feedback control system (43) under control (51) with the packet dropout rate $\lambda$. If there exist matrices $N=N^{T}>0$ and $K_{X}$, such that

$$
\left[\begin{array}{cc}
-N & E\left[\left(\tilde{A}+\tilde{B} K_{X}\right)\right] \\
E\left[\left(\tilde{A}+\tilde{B} K_{X}\right)\right]^{T} & -N^{-1}
\end{array}\right]<0,
$$




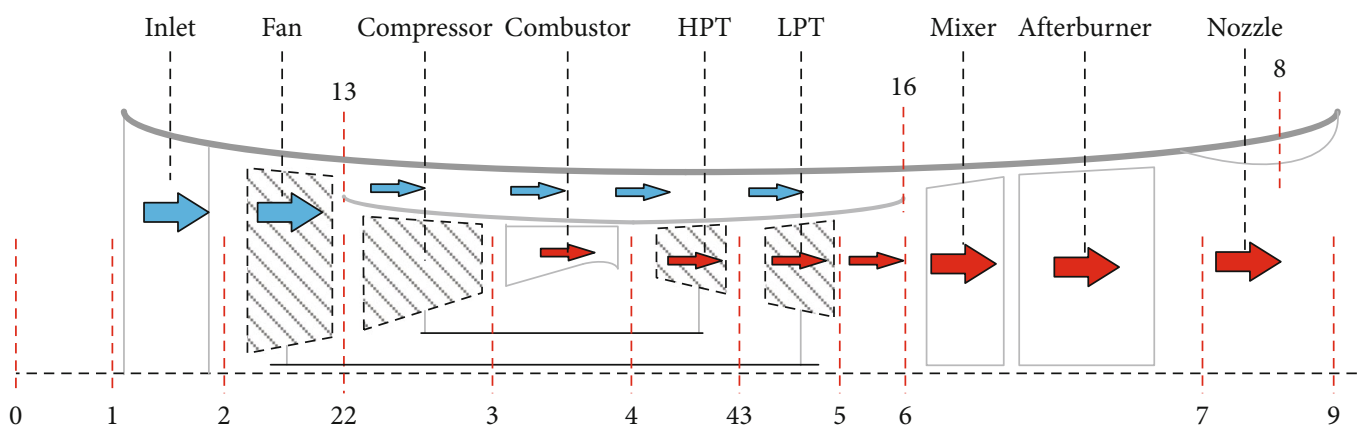

Figure 6: Diagram of a two-spool turbofan engine.

where

$E\left[\left(\tilde{A}+\tilde{B} K_{X}\right)\right]=\left[\begin{array}{cc}(1-\lambda)^{2} & (1-\lambda) \lambda \\ \lambda & 0\end{array}\right] A+\left[\begin{array}{cc}(1-\lambda)^{2} & (1-\lambda) \lambda \\ \lambda(1-\lambda) & \lambda^{2}\end{array}\right] B K_{X}$,

controlled system (52) is asymptotically stable.

Proof. Consider the Lyapunov function candidate

$$
\begin{gathered}
V\left(\tilde{x}_{k}\right)=E\left[\tilde{x}_{k}\right]^{T} N E\left[\tilde{x}_{k}\right], \\
V\left(\tilde{x}_{k+1}\right)=E\left[\tilde{x}_{k+1}\right]^{T} N E\left[\tilde{x}_{k+1}\right],
\end{gathered}
$$

The deviation of $V\left(\tilde{x}_{k+1}\right)$ along the time trajectory of the system (43) under control (51) is

$$
\begin{aligned}
\Delta V\left(\tilde{x}_{k+1}\right)= & V\left(\tilde{x}_{k+1}\right)-V\left(\tilde{x}_{k}\right)=\tilde{x}_{k}^{T} E\left[\left(\tilde{A}+\tilde{B} K_{X}\right)\right]^{T} N E \\
& \cdot\left[\left(\tilde{A}+\tilde{B} K_{X}\right)\right] \tilde{x}_{k}+\tilde{x}_{k}^{T} E\left[\left(\tilde{A}+\tilde{B} K_{X}\right)\right]^{T} N E\left[\tilde{w}_{k}\right] \\
& +E\left[\tilde{w}_{k}\right]^{T} N E\left[\left(\tilde{A}+\tilde{B} K_{X}\right)\right] \tilde{x}_{k}+E\left[\tilde{w}_{k}\right]^{T} N E\left[\tilde{w}_{k}\right] \\
& -E\left[\tilde{x}_{k}\right]^{T} N E\left[\tilde{x}_{k}\right] .
\end{aligned}
$$

By (4), equation (60) can be simplified as

$$
\Delta V\left(\tilde{x}_{k+1}\right)=\tilde{x}_{k}^{T}\left(E\left[\left(\tilde{A}+\tilde{B} K_{X}\right)\right]^{T} N E\left[\left(\tilde{A}+\tilde{B} K_{X}\right)\right]-N\right) \tilde{x}_{k} .
$$

According to Lemma 12 and formula (56), we have

$$
E\left[\left(\tilde{A}+\tilde{B} K_{X}\right)\right]^{T} N E\left[\left(\tilde{A}+\tilde{B} K_{X}\right)\right]-N<0 .
$$

Therefore, $\Delta V\left(\tilde{x}_{k+1}\right)<0$. Theorem 14 is proved.

Remark 15. In the proof of Theorem 14, the Lyapunov function candidates (58)-(59) are designed with mathematical expectation $E[\bullet]$ of the hybrid system states $\tilde{x}_{k}$ and $\tilde{x}_{k+1}$, which introduce the packet dropout rate $\lambda$. Hence, stability condition (56) is dropout dependent.

Remark 16. Due to the introduction of the network in the DCS, the packet dropout generates consequentially and causes the performance deterioration of the controlled system. To deal with this problem, a new framework consisting of the missing information compensation and the dropoutdependent control is proposed. The packet dropout is modelled as (6) and a HAKF approach (28)-(32) estimates the missing states. A dropout-dependent control design with stability condition (56) is presented based on the estimated information. The closed-loop control system is proved stable with the packet dropout.

\section{Application to Turbofan Engine}

Consider a two-spool turbofan engine shown in Figure 6 that is described as (3) with

$$
\left\{\begin{array}{l}
x_{k}=\left[n_{L} n_{H}\right]_{k}^{T}, u_{k}=\left[\begin{array}{ll}
W_{f} A_{8}
\end{array}\right]_{k}^{T}, \\
y_{k}=\left[n_{L} n_{H}\right]_{k}^{T}, z_{k}=\left[\begin{array}{llll}
T_{\mathrm{t} 3} & P_{t 3} & T_{t 6} & P_{t 6}
\end{array}\right]_{k}^{T},
\end{array}\right.
$$

where $n_{L}$ and $n_{H}$ are the low-pressure (LP) rotor speed and the high-pressure (HP) rotor speed, $W_{f}$ is the main fuel flow, $A_{8}$ is the nozzle throat area, $T_{\mathrm{t} 3}$ and $P_{t 3}$ are the total temperature and pressure at the outlet of the compressor, and $T_{\mathrm{t} 6}$ and $P_{\mathrm{t} 6}$ are the total temperature and total pressure at the outlet of LP turbine.

At the typical operation point $(H=0 \mathrm{~km}, M a=0)$ in the flight envelope, the resulted matrices are

$$
\left\{\begin{array}{c}
A=\left[\begin{array}{cc}
0.929 & -0.07 \\
-0.046 & -0.953
\end{array}\right], B=\left[\begin{array}{ll}
0.052 & 0.049 \\
0.034 & 0.032
\end{array}\right], \\
C=I_{2 \times 2}, D=0_{2 \times 2}, W=\left[\begin{array}{cc}
0.118 & 0.252 \\
0.171 & 0.619 \\
0.045 & -0.258 \\
0.574 & -0.005
\end{array}\right],
\end{array}\right.
$$

where $I_{2 \times 2}$ is an identify matrix and $0_{2 \times 2}$ is a zero matrix. 


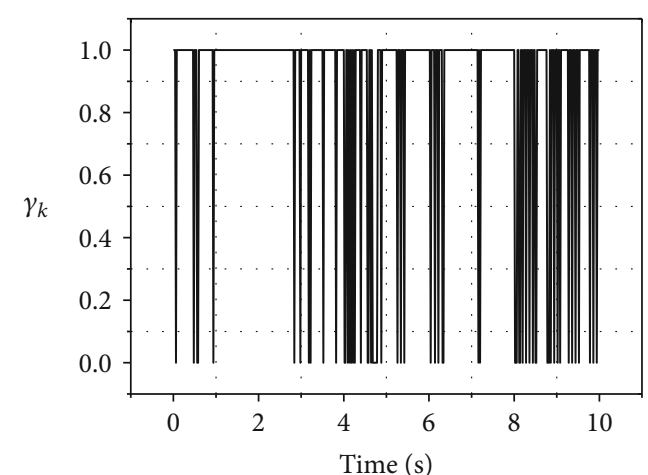

Figure 7: Packet dropout history with $\lambda=10 \%$.

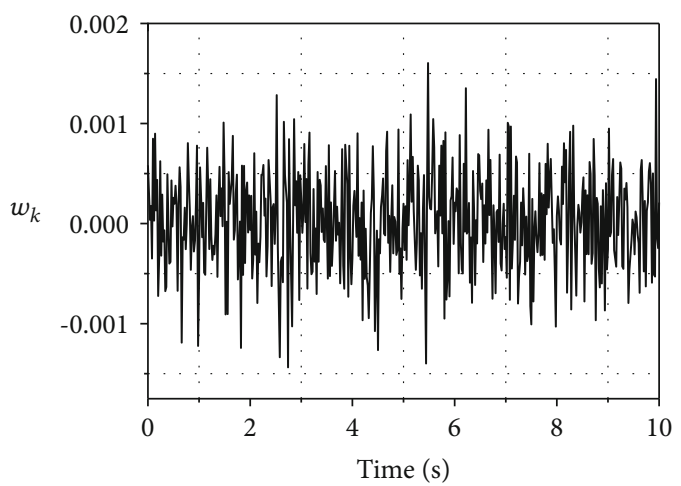

FIGURE 8: System noise.

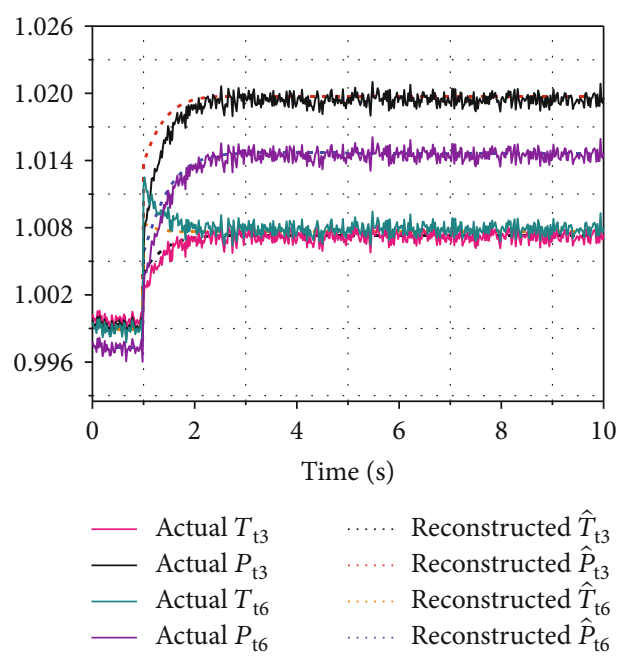

Figure 9: Comparison between reconstructed measurements and actual measurements.

Next, we first present the specific implementation process for the simulation, and then, by this process, two cases of simulations are conducted. The process for the simulation can be summarized below.

Step 1. Based on the nonlinear model formulated by thermodynamic characteristics of the turbofan engine, the matrices $A, B, C, D$, and $W$ in equation (3) are obtained by small per-

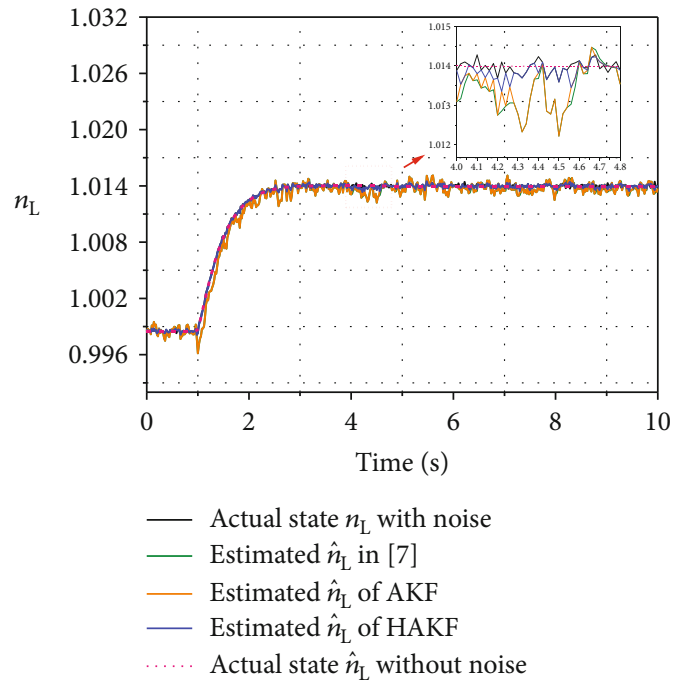

Figure 10: State $n_{L}$ and its estimations of three methods.

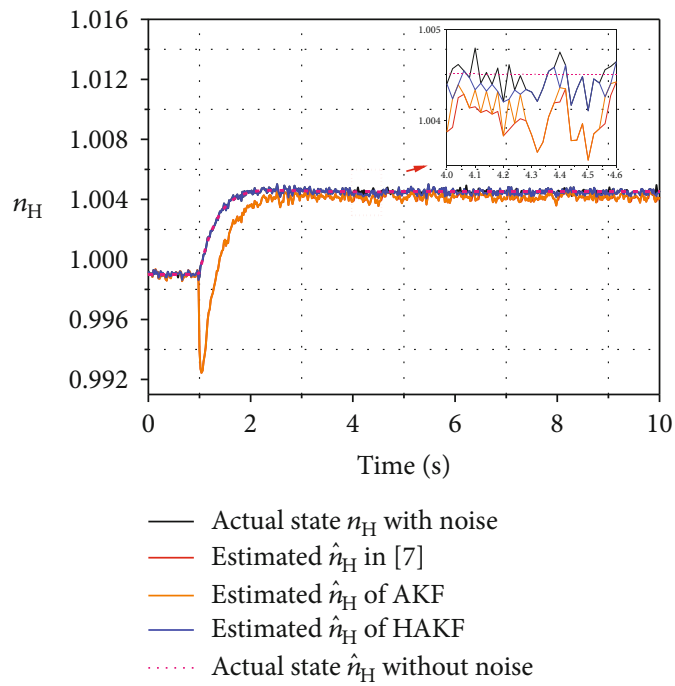

FIGURE 11: State $n_{H}$ and its estimations of three methods.

turbation methods $[43,44]$ at the typical operating point $H$ $=0 \mathrm{~km}, M a=0$.

Step 2. Input the packet dropout rate $\lambda$ for the simulation and then calculate the state feedback controller $K_{X}$ by the LMI (56).

Step 3. Set up the initial state values for $\widehat{x}_{0}, u_{0}$, and $\widehat{P}_{0}$ and the simulation noise with covariance matrix $Q_{k}, R_{k}$.

Step 4. Based on the matrices $A, B, C, D$, and $W$ with the initials, reconstruct the measurements by equations (15)-(16) and then update the estimated state by (28)-(32).

Step 5. The controller $\tilde{u}_{k}$ is obtained by equation (51) and then imports the inputs into the turbofan engine. 


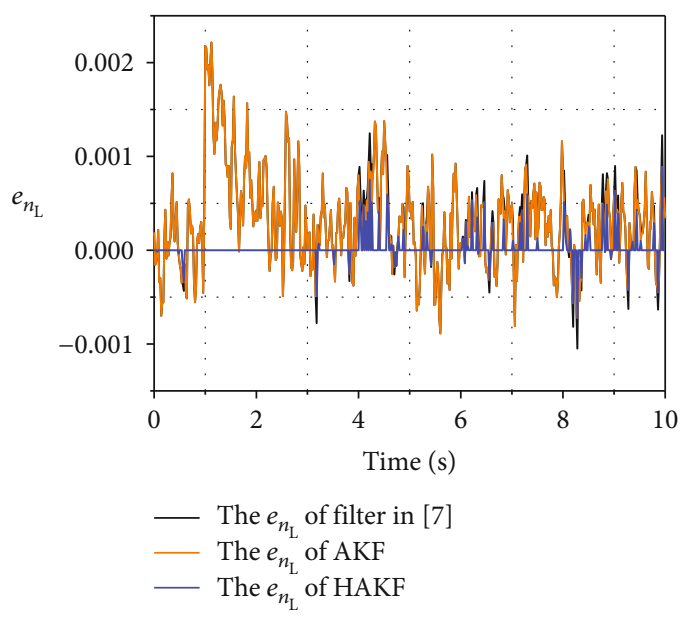

FIGURE 12: The estimated error $e_{n_{L}}$.

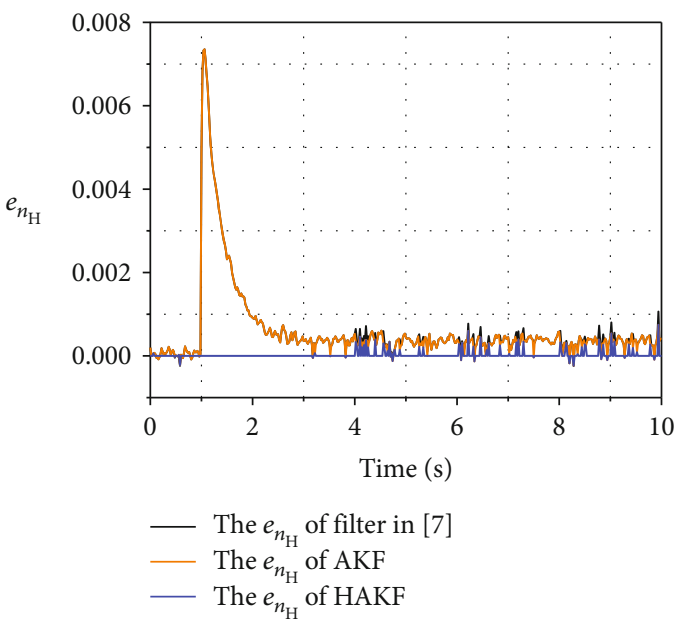

Figure 13: The estimated error $e_{n_{H}}$.

5.1. State Estimation Simulation for Turbofan Engines with Packet Dropout. We first verify the estimation performance of the HAKF by simulation under the operation condition $H=0 \mathrm{~km}, M a=0$. The packet dropout is a Bernoulli process with $\lambda=10 \%$ and the initials are given as

$$
x_{0}=\left[\begin{array}{ll}
1 & 1
\end{array}\right]^{T}, P_{0}=Q_{0}=0.001 I_{2 \times 2}, R_{0}=0.001 I_{4 \times 4} \text {. }
$$

By equations (16) and (28)-(32), the reconstructed measurements and the estimated states are, respectively, obtained with the presence of packet dropout. Figure 7 shows packet dropout history by the variable $\gamma_{k}$. In the figure, " 1 " indicates the successful measurements receive of estimator. On the contrary, "0" suggests the packets dropout. Figure 8 gives the noise history. Figure 9 shows the comparison between reconstructed measurements and its actual value. It is clearly to find that the reconstructed measurements can track the actual quickly and precisely, which guarantees the accuracy of HAKF.

Figures 10 and 11 show the histories of estimated states $\widehat{n}_{L}, \widehat{n}_{H}$ and their true values $n_{L}, n_{H}$. We can discover that

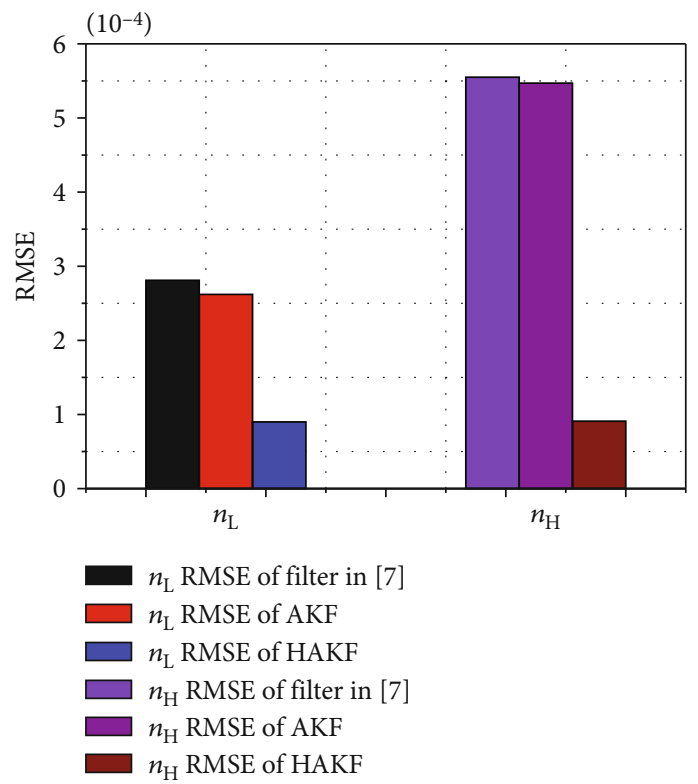

FiguRE 14: RMSEs of three estimation methods.

the estimation states of HAKF (blue line) are close to the actual value even at the packet dropout instant. Figures 12 and 13 further give the estimation errors of $n_{L}$ and $n_{H}$. The RMSEs of $n_{L}$ and $n_{H}$ by the HAKF are $0.901 \times 10^{-4}$ and $0.91 \times 10^{-4}$, which are shown in Figure 14 .

To compare the new estimator with the traditional Kalman estimation approach shown in [7], the adaptive Kalman filter (AKF) is conducted under the same simulation conditions as the HAKF. The simulation results of these two approaches are also depicted in Figures 10-14. From Figures 10 and 11, we can find that the filter in [7] has bigger jitter than the HAKF at the steady state. There is a significant droop at $1 \mathrm{~s}$ resulted by the step of inputs when the aeroengine transits to another steady state. The measurements vary sharply in Figure 9, which cause the significant droop in Figures 10 and 11. By Figure 14, we can find that, comparing with the filter in [7] and the AKF, the RSMEs of the new filter are reduced by $67.95 \%$ and $65.55 \%$ for $n_{L}$ and by $83.61 \%$ and $83.35 \%$ for $n_{H}$. It demonstrates that, because of the collaboration of the measurements and their reconstructions when packet dropout, the estimation accuracy of HAKF is significantly superior.

Furthermore, we conducted the simulation under more different operation conditions with different packet dropout rate $\lambda$ in the flight envelope. The RMSEs of the two states of three estimation methods are listed in Tables 1 and 2. The results in Tables 1 and 2 show that the RMSEs of HAKF decreases over 57\% compared to the traditional Kalman filter in [7] at all simulation conditions, and the maximum error reduction is $83.61 \%$. It can be intuitively concluded that the HAKF has the significant improvement in estimation accuracy compared to the traditional Kalman filter.

5.2. Stability Simulation for the Controlled Turbofan Engines with Hybrid Signals. In this subsection, we performed the simulation to verify the stability of the controlled turbofan 
TABLE 1: $n_{L}$ RMSE of three methods.

\begin{tabular}{lccccc}
\hline $\begin{array}{l}\text { Operation } \\
\text { points }\end{array}$ & $\lambda$ & $\begin{array}{c}\text { Rilter in } \\
\text { [7] }\end{array}$ & AKF & HAKF & $\begin{array}{c}\text { Decrease to [7] } \\
(\%)\end{array}$ \\
\hline$H=0 \mathrm{~km}$ & $10 \%$ & 2.81 & 2.62 & 0.90 & -67.95 \\
$M a=0$ & $20 \%$ & 2.82 & 2.60 & 0.82 & -71.11 \\
& $30 \%$ & 2.89 & 2.42 & 1.22 & -57.74 \\
$H=4 \mathrm{~km}$ & $10 \%$ & 4.86 & 4.48 & 1.57 & -67.63 \\
$M a=0.4$ & $20 \%$ & 4.33 & 3.99 & 1.30 & -69.98 \\
& $30 \%$ & 4.38 & 3.81 & 1.83 & -58.24 \\
$H=6 \mathrm{~km}$ & $10 \%$ & 3.28 & 3.13 & 0.79 & -75.82 \\
$M a=0.8$ & $20 \%$ & 3.16 & 2.95 & 0.77 & -75.77 \\
& $30 \%$ & 3.00 & 2.72 & 1.06 & -64.55 \\
$H=10 \mathrm{~km}$ & $10 \%$ & 5.49 & 5.22 & 1.21 & -77.90 \\
$M a=1$ & $20 \%$ & 4.72 & 4.28 & 0.93 & -80.19 \\
& $30 \%$ & 4.87 & 4.29 & 1.41 & -71.05 \\
\hline
\end{tabular}

TABLE 2: $n_{H}$ RMSE of three methods.

\begin{tabular}{lccccc}
\hline $\begin{array}{l}\text { Operation } \\
\text { points }\end{array}$ & $\lambda$ & $\begin{array}{c}\text { Rilter in } \\
{[7]}\end{array}$ & AKF & HAKF & $\begin{array}{c}\text { Decrease to [7] } \\
(\%)\end{array}$ \\
\hline$H=0 \mathrm{~km}$ & $10 \%$ & 5.55 & 5.47 & 0.91 & -83.61 \\
$M a=0$ & $20 \%$ & 5.39 & 5.29 & 1.07 & -80.10 \\
& $30 \%$ & 4.96 & 4.79 & 1.29 & -74.05 \\
$H=4 \mathrm{~km}$ & $10 \%$ & 3.91 & 3.61 & 1.14 & -70.82 \\
$M a=0.4$ & $20 \%$ & 3.74 & 3.48 & 1.02 & -72.66 \\
& $30 \%$ & 3.52 & 3.14 & 1.33 & -62.31 \\
$H=6 \mathrm{~km}$ & $10 \%$ & 3.02 & 3.03 & 0.94 & -68.93 \\
$M a=0.8$ & $20 \%$ & 2.99 & 3.01 & 1.08 & -63.82 \\
& $30 \%$ & 2.99 & 3.02 & 1.47 & -50.80 \\
$H=10 \mathrm{~km}$ & $10 \%$ & 8.15 & 7.99 & 1.71 & -79.03 \\
$M a=1$ & $20 \%$ & 8.12 & 7.88 & 2.01 & -75.20 \\
& $30 \%$ & 7.68 & 7.30 & 2.54 & -66.99 \\
\hline
\end{tabular}

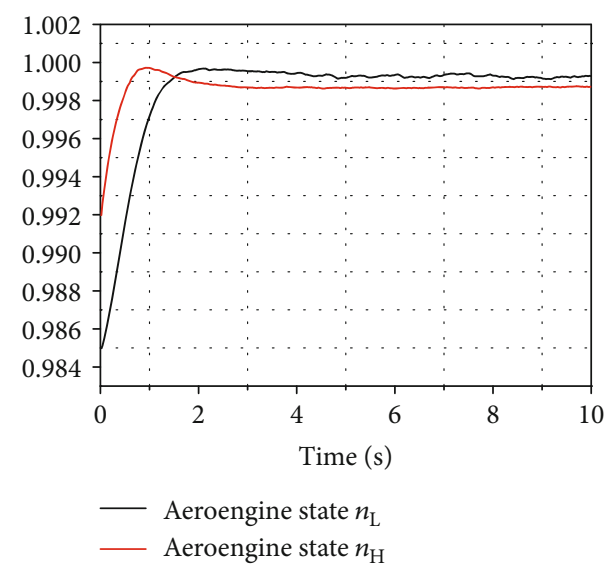

Figure 15: States $n_{L}$ and $n_{H}$ stabilization history of control system with $\lambda=10 \%$.

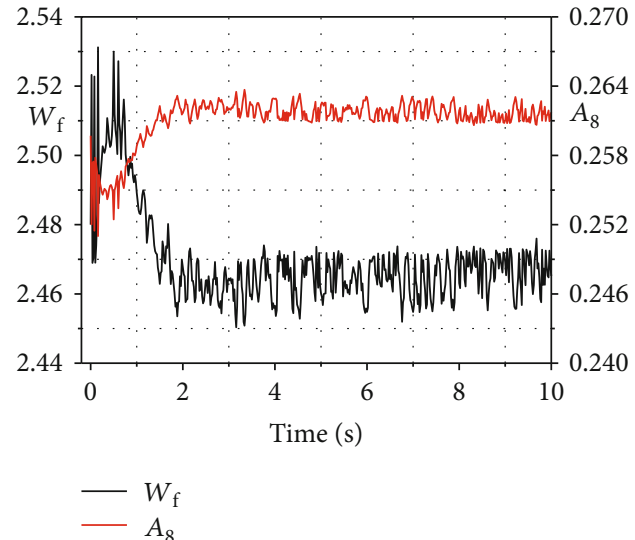

Figure 16: Histories of control inputs $W_{f}$ and $A_{8}$.

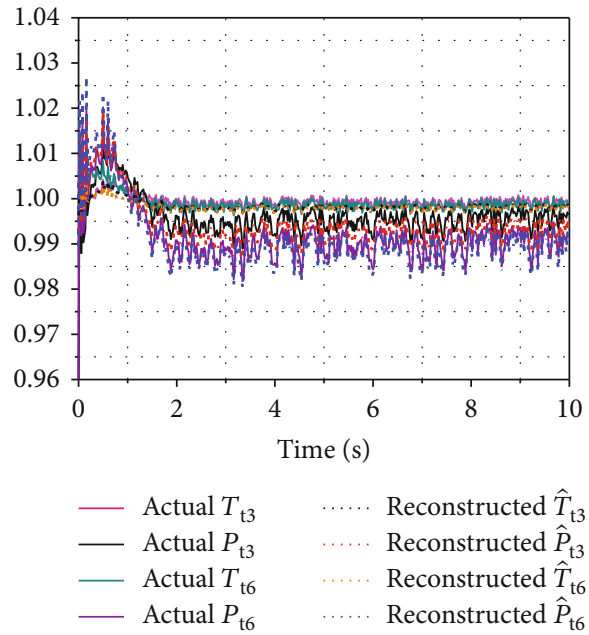

FIGURE 17: Measurements and their reconstructed values.

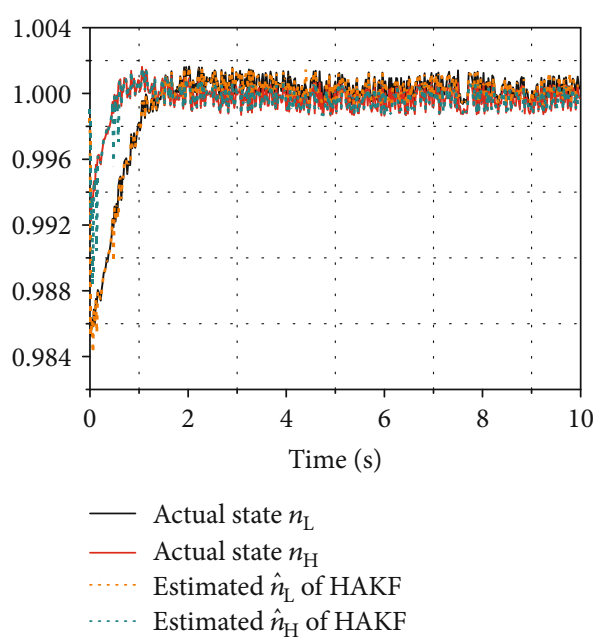

FIGURE 18: States and their estimations by HAKF. 
system (3), (63), and (64) with HAKF (28)-(32) and the state feedback controller (51).

Remark 17. It is worth noting that the stability simulation is also conducted at the typical operation point $(H=0 \mathrm{~km}, M$ $a=0)$. The system mathematical description is same with (64) and the simulation contained the HAKF estimation module under packet dropout.

By Theorem 14, solving the LMI (56), the control gain of (51) was obtained as

$$
K_{X}=\left[\begin{array}{cccc}
-3.725 & -0.455 & -0.412 & -0.053 \\
-0.455 & -13.211 & -0.064 & -1.467 \\
-0.412 & -0.064 & -0.0004 & 0.029 \\
-0.053 & -1.467 & 0.029 & -0.001
\end{array}\right]
$$

The simulation results are displayed in Figures 15-18. Figure 15 demonstrates the aeroengine states with $\lambda=10 \%$. By Figure 15, we can observe that the states can converge to the equilibrium points $x_{0}=\left[\begin{array}{ll}0.9993 & 0.9987\end{array}\right]^{T}$ after $2 \mathrm{~s}$, which means this controlled turbofan with a hybrid signal system is stable under the packet dropout. The control inputs are shown in Figure 16. The measurement reconstruction and estimation of HAKF are depicted in Figures 17 and 18. Figure 16 shows that the inputs $W_{f}$ and $A_{8}$ jitter due to the presence of packet dropout and noises. We can find that, in Figure 17, the measurement reconstruction has a good recovering ability to the missing measurements, and in Figure 18, the HAKF has excellent estimation accuracy.

\section{Conclusions}

For controlling the aeroengine with distributed control system framework, there is a challenge, the packet dropout, which should be investigated. The literature researches focus on the estimation for packet dropout or the robust control that covering packet dropout independently. We proposed an estimation-based and dropout-dependent control approach. The estimation is first concerned about the measurement reconstruction by an inverse system combined with references. Then, a hybrid adaptive Kalman filter is proposed to estimate the missing states with the assistance of reconstructed measurements, and the stability of this estimation approach is proved to be specific and sufficient. Finally, a simply and intuitively design method for the estimation-based and dropout-dependent control is proposed. The novel control scheme is applied to a turbofan engine. The simulations demonstrate that under the same simulation conditions, comparing to the estimation approach in [7], RSMEs of HAKF is reduced by $67.95 \%$ for $n_{L}$ and by $83.61 \%$ for $n_{H}$ which directly and clearly shows that the HAKF has significant estimation accuracy advantage. It can be concluded that the HAKF has satisfactory and effective ability (effectiveness) for compensating missing states. And the stability of a turbofan engine controlled sys- tem is guaranteed and verified by the simulation. This paper presents a novel estimation in combination with state feedback control for the aeroengine distributed control system with network packet dropout.

\section{Data Availability}

The data used to support the research of this paper are available from the corresponding author upon reasonable request.

\section{Conflicts of Interest}

The authors declare that there is no conflict of interest regarding the publication of this paper.

\section{Acknowledgments}

This work was supported by the National Science and Technology Major Project (No. 2019-V-0003-0094) and China Fundamental Research Funds for Chinese Central Universities (No. 300102259306).

\section{References}

[1] M. X. Pan, L. J. Cao, W. X. Zhou, J. Q. Huang, and Y. H. Chen, "Robust decentralized control design for aircraft engines: a fractional type," Chinese Journal of Aeronautics, vol. 32, no. 2, pp. 347-360, 2019.

[2] R. K. Yedavalli, M. S. Sabatto, and A. Behbahani, "Framework for distributed engine control system for sampled-data systems with uncertain time-varying sampling intervals and delays with state estimations," in 51st AIAA/SAE/ASEE Joint Propulsion Conference, p. 3990, Orlando, United states, 2015.

[3] K. Plarre and F. Bullo, "On Kalman filtering for detectable systems with intermittent observations," IEEE Transactions on Automatic Control, vol. 54, no. 2, pp. 386-390, 2009.

[4] H. M. Qian, Z. B. Qiu, and Y. H. Wu, "Robust extended Kalman filtering for nonlinear stochastic systems with random sensor delays, packet dropouts and correlated noises," Aerospace Science and Technology, vol. 66, pp. 249-261, 2017.

[5] E. N. Gilbert, "Capacity of a burst-noise channel," Bell System Technical Journal, vol. 39, no. 5, pp. 1253-1265, 1960.

[6] M. Mushkin and I. Bar-David, "Capacity and coding for the Gilbert-Elliott channels," IEEE Transactions on Information Theory, vol. 35, no. 6, pp. 1277-1290, 1989.

[7] B. Sinopoli, L. Schenato, M. Franceschetti, K. Poolla, M. I. Jordan, and S. S. Sastry, "Kalman filtering with intermittent observations," IEEE Transactions on Automatic Control, vol. 49, no. 9, pp. 1453-1464, 2004.

[8] M. Y. Huang and S. Dey, "Stability of Kalman filtering with Markovian packet losses," Automatica, vol. 43, no. 4, pp. 598-607, 2007.

[9] J. F. Wu, L. Shi, L. H. Xie, and K. H. Johansson, “An improved stability condition for Kalman filtering with bounded Markovian packet losses," Automatica, vol. 62, pp. 32-38, 2015.

[10] L. Xie and L. H. Xie, "Stability of a random Riccati equation with Markovian binary switching," IEEE Transactions on Automatic Control, vol. 53, no. 7, pp. 1759-1764, 2008.

[11] J. F. Wu, G. D. Shi, B. D. Anderson, and K. H. Johansson, "Kalman filtering over Gilbert-Elliott channels: stability conditions 
and critical curve," IEEE Transactions on Automatic Control, vol. 63, no. 4, pp. 1003-1017, 2018.

[12] O. Thapliyal, J. S. Nandiganahalli, and I. Hwang, "Kalman filtering with state-dependent packet losses," IET Control Theory and Applications, vol. 13, no. 2, pp. 306-312, 2019.

[13] H. J. Yang, H. Li, Y. Q. Xia, and L. Li, "Nonuniform sampling Kalman filter for networked systems with Markovian packets dropout," Journal of the Franklin Institute, vol. 355, no. 10, pp. 4218-4240, 2018.

[14] D. Marelli, T. J. Sui, and M. Y. Fu, "Stability analysis for Kalman filters with random measurement matrices," in 2017 13th IEEE International Conference on Control \& Automation (ICCA), pp. 502-506, Ohrid, Macedonia, 2017.

[15] D. Marelli, T. J. Sui, E. Rohr, and M. Y. Fu, "Stability of Kalman filtering with a random measurement equation: application to sensor scheduling with intermittent observations," Automatica, vol. 99, pp. 390-402, 2019.

[16] S. W. Gao, P. P. Chen, D. Huang, and Q. Niu, "Stability analysis of multi-sensor Kalman filtering over lossy networks," Sensors, vol. 16, no. 4, p. 566, 2016.

[17] H. Ishii, " $H_{\infty}$ control with limited communication and message losses," Systems and Control Letters, vol. 57, no. 4, pp. 322-331, 2008.

[18] H. Y. Cheng, C. Y. Dong, W. L. Jiang, Q. Wang, and Y. Z. Hou, "Non-fragile switched $H_{\infty}$ control for morphing aircraft with asynchronous switching," Chinese Journal of Aeronautics, vol. 30, no. 3, pp. 1127-1139, 2017.

[19] A. W. A. Saif, "New results on the observer-based $H_{\infty}$ control for uncertain nonlinear networked control systems with random packet losses," IEEE Access, vol. 7, pp. 26179-26191, 2019.

[20] E. M. Asl, F. Hashemzadeh, M. Baradarannia, and P. Bagheri, "Observer-based controller design for a class of networked control systems with transmission delays and packet losses," in 2021 7th International Conference on Control, Instrumentation and Automation (ICCIA), pp. 1-6, Tabriz, Iran, 2021.

[21] A. W. A. Saif, "Resilient observer-based $H_{\infty}$ control of uncertain nonlinear networked control systems," IEEE Access, vol. 9, pp. 104636-104652, 2021.

[22] X. L. Shao, Y. Shi, and W. D. Zhang, "Input-and-measurement event-triggered output-feedback chattering reduction control for MEMS gyroscopes," IEEE Transactions on Systems, Man, and Cybernetics: Systems, pp. 1-12, 2021.

[23] X. L. Shao, Y. Shi, and W. D. Zhang, "Fault-tolerant quantized control for flexible air-breathing hypersonic vehicles with appointed-time tracking performances," IEEE Transactions on Aerospace and Electronic Systems, vol. 57, no. 2, pp. 12611273, 2021.

[24] X. H. Yue, X. L. Shao, and W. D. Zhang, "Elliptical encircling of quadrotors for a dynamic target subject to aperiodic signals updating," IEEE Transactions on Intelligent Transportation Systems, pp. 1-14, 2021.

[25] Z. D. Wang, F. W. Yang, D. W. Ho, and X. H. Liu, "Robust $H_{\infty}$ control for networked systems with random packet losses," IEEE Transactions on Systems, Man, and Cybernetics, Part B (Cybernetics), vol. 37, no. 4, pp. 916-924, 2007.

[26] J. G. Li, J. Q. Yuan, and J. G. Lu, "Observer-based H(infinity) control for networked nonlinear systems with random packet losses," ISA Transactions, vol. 49, no. 1, pp. 39-46, 2010.

[27] Z. M. Li and X. H. Chang, "Robust $\mathrm{H}<\mathrm{sub}>\infty</$ sub $>$ control for networked control systems with randomly occurring uncertainties: observer-based case," ISA Transactions, vol. 83, pp. 13-24, 2018.

[28] W. H. Qi, Y. K. Hou, G. D. Zong, and C. K. Ahn, "Finite-time event-triggered control for semi-markovian switching cyberphysical systems with FDI attacks and applications," IEEE Transactions on Circuits and Systems I: Regular Papers, vol. 68, no. 6, pp. 2665-2674, 2021.

[29] W. H. Qi, G. D. Zong, and W. X. Zheng, "Adaptive eventtriggered SMC for stochastic switching systems with semiMarkov process and application to boost converter circuit model," IEEE Transactions on Circuits and Systems I: Regular Papers, vol. 68, no. 2, pp. 786-796, 2021.

[30] Y. J. Jin, W. H. Qi, and G. D. Zong, "Finite-time synchronization of delayed semi-Markov neural networks with dynamic event-triggered scheme," International Journal of Control, Automation and Systems, vol. 19, no. 6, pp. 2297-2308, 2021.

[31] X. Wang and S. L. Sun, "Optimal recursive estimation for networked descriptor systems with packet dropouts, multiplicative noises and correlated noises," Aerospace Science and Technology, vol. 63, pp. 41-53, 2017.

[32] L. Su and G. Chesi, "Robust stability of uncertain linear systems with input and output quantization and packet loss," Automatica, vol. 87, pp. 267-273, 2018.

[33] M. Xue, H. C. Yan, H. Zhao, H. Shen, and P. Shi, "Dissipativity-based filter design for Markov jump systems with packet loss compensation," Automatica, vol. 133, article 109843, 2021.

[34] G. A. Terejanu, Discrete Kalman filter tutorial, vol. 14260, University at Buffalo, Department of Computer Science and Engineering, NY, 2013.

[35] H. Yao, Full Authority Digital Electronic Control System for Aero-Engine, Aviation industry press, Beijing, China, 2014.

[36] X. X. Feng, S. H. Li, and F. Pan, "State estimate for stochastic systems with dual unknown interference inputs," Chinese Journal of Aeronautics, vol. 33, no. 9, pp. 2395-2407, 2020.

[37] B. Gary and G. Welch, "An introduction to the Kalman filter," SIGGRAPH, p. 41, 2001.

[38] F. Lu, T. Y. Y. Gao, J. Q. Huang, and X. J. Qiu, "Nonlinear Kalman filters for aircraft engine gas path health estimation with measurement uncertainty," Aerospace Science and Technology, vol. 76, pp. 126-140, 2018.

[39] J. X. Feng, Z. D. Wang, and M. Zeng, "Optimal robust nonfragile Kalman-type recursive filtering with finite-step autocorrelated noises and multiple packet dropouts," Aerospace Science and Technology, vol. 15, no. 6, pp. 486-494, 2011.

[40] J. L. Crassidis and J. L. Junkins, Optimal Estimation of Dynamic Systems, CRC press, Boca Raton, Florida, USA, 2011.

[41] T. Karvonen, Stability of linear and non-linear Kalman filters, Master's thesis, University of Helsinki, 2014.

[42] B. X. Hu, Q. K. Song, and Z. J. Zhao, "Robust state estimation for fractional-order complex-valued delayed neural networks with interval parameter uncertainties: LMI approach," Applied Mathematics and Computation, vol. 373, article 125033, 2020.

[43] W. H. Xu, J. Q. Huang, J. K. Qin, and M. X. Pan, "Limit protection design in turbofan engine acceleration control based on scheduling command governor," Chinese Journal of Aeronautics, vol. 34, no. 10, pp. 67-80, 2021.

[44] F. Lu, J. N. Qian, J. Q. Huang, and X. J. Qiu, "In-flight adaptive modeling using polynomial LPV approach for turbofan engine dynamic behavior," Aerospace Science and Technology, vol. 64, pp. 223-236, 2017. 